\title{
Seroprevalence of Anti-SARS-CoV-2 Antibodies in Blood Donors from Nuevo Leon State, Mexico, during 2020: A Retrospective Cross-Sectional Evaluation
}

\author{
Natalia Martinez-Acuña 1,2, Diana Minerva Avalos-Nolazco ${ }^{1}{ }^{\mathbb{D}}$, Diana Raquel Rodriguez-Rodriguez ${ }^{1}$, \\ Cynthia Gabriela Martinez-Liu 1,2 , Kame Alberto Galan-Huerta 1,2 (D), Gerardo Raymundo Padilla-Rivas ${ }^{1}$ (D), \\ Javier Ramos-Jimenez ${ }^{2,3}$, Sergio Ayala-de-la-Cruz ${ }^{4}$, Eduardo Cienfuegos-Pecina 4 (D), Erik Alejandro Diaz-Chuc ${ }^{4}$, \\ Rogelio Cazares-Tamez ${ }^{4}$, Amador Flores-Arechiga ${ }^{4}$, Fernando Perez-Chavez 4 , Daniel Arellanos-Soto ${ }^{1,2}$ (D), \\ Sonia Amelia Lozano-Sepulveda ${ }^{1,2}$, Elvira Garza-Gonzalez ${ }^{1}$, Consuelo Treviño-Garza ${ }^{5,6}$, \\ Roberto Montes-de-Oca-Luna ${ }^{6,7}$ (i) , Aurora Beatriz Lee-Gonzalez ${ }^{6,8}$, Manuel Enrique de-la-O-Cavazos ${ }^{5,6}$ \\ and Ana Maria Rivas-Estilla 1,2,*(D)
}

\section{check for} updates

Citation: Martinez-Acuña, N.; Avalos-Nolazco, D.M.

Rodriguez-Rodriguez, D.R.;

Martinez-Liu, C.G.; Galan-Huerta, K.A.; Padilla-Rivas, G.R.;

Ramos-Jimenez, J.; Ayala-de-la-Cruz S.; Cienfuegos-Pecina, E.; Diaz-Chuc, E.A.; et al. Seroprevalence of Anti-SARS-CoV-2 Antibodies in Blood Donors from Nuevo Leon State, Mexico, during 2020: A Retrospective Cross-Sectional Evaluation. Viruses 2021, 13, 1225. https://doi.org/ $10.3390 / v 13071225$

Academic Editors: Eurico Arruda, Helena Lage Ferreira, José Luiz Proença Módena and William Marciel de Souza

Received: 16 April 2021 Accepted: 15 June 2021 Published: 24 June 2021

Publisher's Note: MDPI stays neutral with regard to jurisdictional claims in published maps and institutional affiliations.

Copyright: (C) 2021 by the authors. Licensee MDPI, Basel, Switzerland. This article is an open access article distributed under the terms and conditions of the Creative Commons Attribution (CC BY) license (https:// creativecommons.org/licenses/by/ $4.0 /)$.
1 Department of Biochemistry and Molecular Medicine, School of Medicine, Autonomous University of Nuevo León, Monterrey 64460, Mexico; nmartinez.me0120@uanl.edu.mx (N.M.-A.); dianaxavalos@gmail.com (D.M.A.-N.); raquelrodriguez85@gmail.com (D.R.R.-R.); cmliu@outlook.es (C.G.M.-L.); kame.galanhr@uanl.edu.mx (K.A.G.-H.); gpadillarivas@gmail.com (G.R.P.-R.); d_arellanos_s@yahoo.com (D.A.-S.); lozano_sonia@hotmail.com (S.A.L.-S.); elvira.garzagn@uanl.edu.mx (E.G.-G.)

2 Center of Research and Innovation on Medical Virology, School of Medicine, Autonomous University of Nuevo Leon, Monterrey 64460, Mexico; javramos31@gmail.com

3 Department of Internal Medicine, Infectious Disease Service, Hospital Universitario "Dr. Jose E. Gonzalez", Autonomous University of Nuevo León, Monterrey 64460, Mexico

4 Department of Clinical Pathology and Blood Transfusion Bank, Hospital Universitario "Dr. Jose E. Gonzalez", Autonomous University of Nuevo León, Monterrey 64460, Mexico;

dr.sergioayala.hu@gmail.com (S.A.-d.-1.-C.); eduardo.cienfuegospe@uanl.edu.mx (E.C.-P.); ediaz.me0085@uanl.edu.mx (E.A.D.-C.); dr.cazares07@gmail.com (R.C.-T.); arechiga@outlook.com (A.F.-A.); fernando.perezchv@uanl.edu.mx (F.P.-C.)

5 Department of Pediatrics, Hospital Universitario “Dr. Jose E. Gonzalez", Autonomous University of Nuevo León, Monterrey 64460, Mexico; cotrevin@hotmail.com (C.T.-G.); delaocavazos@yahoo.com (M.E.d.-1.-O.-C.)

6 Secretariat of Health of Nuevo León State, Monterrey 64460, Mexico; roberto.montesdeocaln@uanl.edu.mx (R.M.-d.-O.-L.); beatriz.lee@saludnl.gob.mx (A.B.L.-G.)

7 Department of Histology, School of Medicine, Hospital Universitario "Dr. Jose E. Gonzalez", Autonomous University of Nuevo León, Monterrey 64460, Mexico

8 Transfusion Center, CETS, Secretariat of Health of Nuevo Leon State, Monterrey 64460, Mexico

* Correspondence: amrivas1@yahoo.ca

Abstract: The progression and distribution of the SARS-CoV-2 pandemic are continuously changing over time and can be traced by blood donors' serological survey. Here, we investigated the seroprevalence of anti-SARS-CoV-2 antibodies in blood donors in Nuevo Leon, Mexico during 2020 as a strategy for the rapid evaluation of the spread of SARS-CoV-2 and asymptomatic case detection. We collected residual plasma samples from blood donors who attended two regional donation centers from January to December of 2020 to identify changes in anti-SARS-CoV-2 IgG prevalence. Plasma samples were analyzed on the Abbott Architect instrument using the commercial Abbott SARS-CoV-2 IgG chemiluminescent assay. We found a total of 99 reactive samples from 2068 analyzed plasma samples, resulting in a raw prevalence of $4.87 \%$. Donors aged $18-49$ years were more likely to be seropositive compared to those aged $>50$ years $(p<0.001)$. Weekly seroprevalence increased from $1.8 \%$ during the early pandemic stage to $27.59 \%$ by the end of the year. Prevalence was 1.46 -fold higher in females compared to males. Case geographical mapping showed that Monterrey city recorded the majority of SARS-CoV-2 cases. These results show that there is a growing trend of seroprevalence over time associated with asymptomatic infection that is unnoticed under the current epidemiological surveillance protocols. 
Keywords: blood donors; COVID-19; anti-SARS-CoV-2 IgG; seroprevalence; Mexico

\section{Introduction}

Severe acute respiratory syndrome coronavirus 2 (SARS-CoV-2), producing coronavirus disease 2019 (COVID-19), has spread worldwide, becoming a significant public health problem. At this time (22 May 2021), it has caused over 166 million cases worldwide and at least 3.4 million deaths [1]. Mexico is one of the most affected countries in Latin America, with 2,392,744 confirmed infections and 221,256 deaths [1]. In the state of Nuevo Leon (Northeast Mexico), the pandemic has caused 177,164 total cases and 10,777 deaths [2]. Nuevo Leon, located at the northern frontier that borders the US state of Texas, hosts one of the three biggest cities in Mexico. Due to its economic activity, which is based on manufacturing, commercial business, and education, $96 \%$ of its 5.6 million inhabitants are concentrated around the urban area of Monterrey [3]. Due to the high population density and the continuous population displacement in Nuevo Leon, as soon as the virus was detected in the region (March 2020), the state closed schools and restricted commercial activities and social meetings. After a series of governmental decisions, Mexico planned a gradual re-opening of its economy in early June 2020, causing an expected increase in the number of COVID-19 cases (Figure 1). The clinical presentation of COVID-19 in patients includes fever, dry cough, and fatigue as the most common symptoms that may appear 3-14 days after infection. Several reports have suggested that SARS-CoV-2 infections can be asymptomatic. It is reported that the asymptomatic infection incidence can range from 1.2 to $12.9 \%$ [4,5] in large populations. However, other studies reported a much higher proportion reaching up to $87.9 \%$ [6,7]. Reports have demonstrated that viral loads are very similar in symptomatic and asymptomatic groups, making the latter capable of spreading SARS-CoV-2 despite the absence of clinical manifestations [8,9]. Mexico has not established a screening protocol to identify asymptomatic cases, but it is necessary to take both symptomatic and asymptomatic infected subjects into account in order to obtain more detailed epidemiologic data and recalculate the prevalence and fatality rates of the COVID-19 pandemic.

Estimating the seroprevalence of anti-SARS-CoV-2 antibodies (Abs) in blood donors is a powerful and cost-effective strategy to monitor the population's exposure and detect asymptomatic SARS-CoV-2 cases. After infection occurs, specific Abs against SARS-CoV-2 appear between four and five days (IgM, immunoglobulin $\mathrm{M}$ ), and most of the patients seroconvert (IgG, immunoglobulin G) within the first three weeks [10-12]. Robust titers of specific Abs can be detected up to five months post-SARS-CoV-2 infection [13].

Early studies focusing on blood donors to identify SARS-CoV-2-infected persons were conducted in different regions $[14,15]$. The seroprevalence of anti-SARS-CoV-2 determined using this approach varies from $0.1 \%$ (San Francisco Bay Area) [16] to 5.6\% (Kenya) but can reach values of up to $9 \%$ when they are adjusted for a specific geographical region [17].

To date, limited data have documented the prevalence of SARS-CoV-2 antibodies in the general population and asymptomatic outpatients in Mexico [18,19]. In this study, we conducted a retrospective cross-sectional seroprevalence survey using residual plasma samples to identify SARS-CoV-2-specific IgG antibodies among blood donors who attended two regional transfusion centers during 2020. The identification of asymptomatic cases of SARS-CoV-2 allowed us to add information to track the progression of the pandemic in our country. 
A
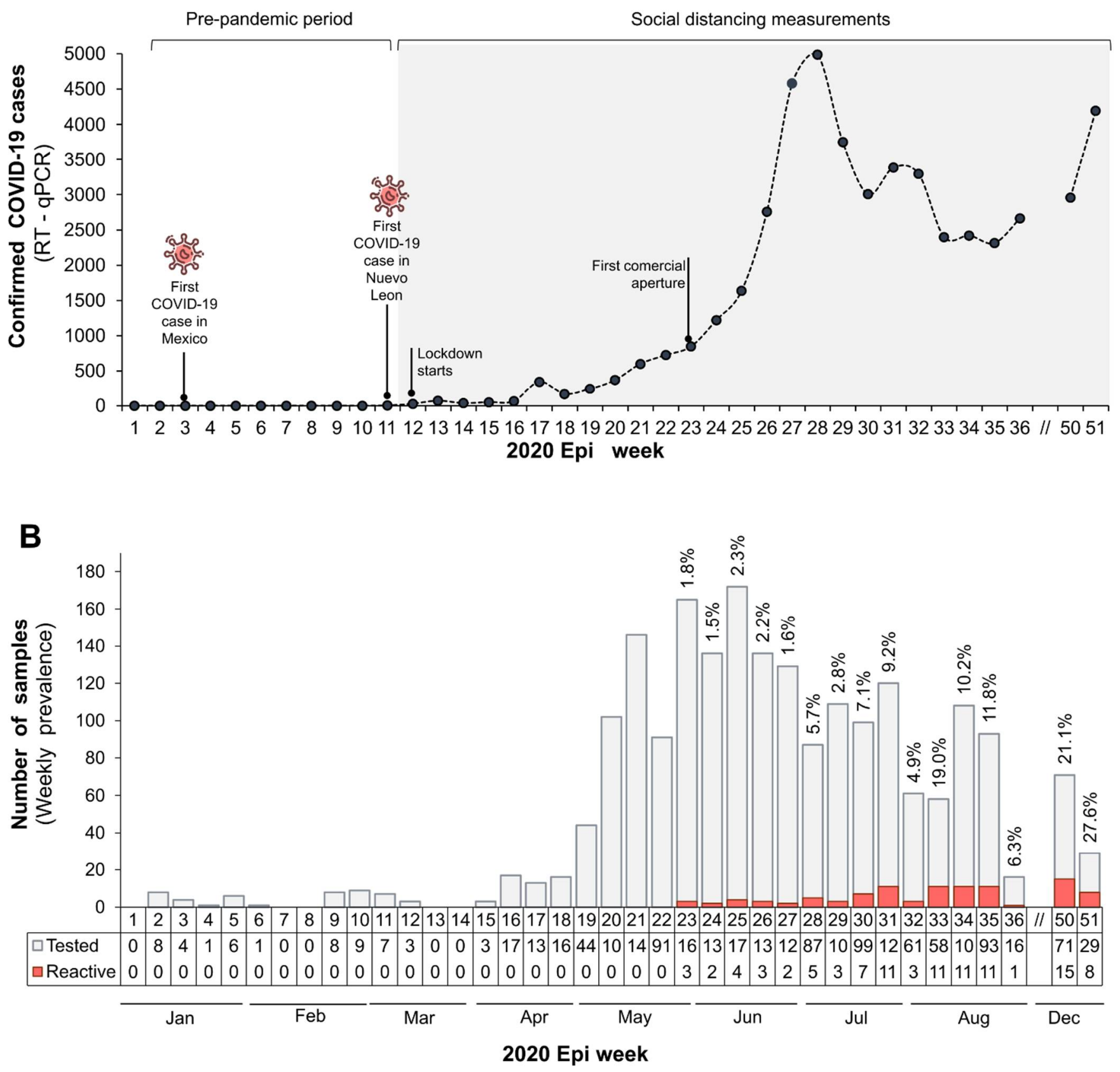

Figure 1. Dynamics of asymptomatic SARS-CoV-2 cases detected by anti-SARS-CoV-2 IgG analysis in blood donors during 2020 in Nuevo Leon, Mexico. (A) Curve of RT-qPCR-confirmed new COVID-19 cases per epi week recorded in Nuevo Leon. The gray shading indicates the pandemic period. Significant pandemic events for the Nuevo Leon area are indicated. (B) Prevalence of anti-SARS-CoV-2 IgG per epi week was estimated by dividing the number of positive cases (red bars) by the number of tested samples (gray bars) per week. S/C index $\geq 1.4$ was considered as a positive result. The respective seroprevalence of SARS-CoV-2 IgG is shown on the top of each bar.

\section{Materials and Methods}

\subsection{Study Design and Subjects}

We performed a retrospective cross-sectional anti-SARS-CoV-2 IgG serological survey study on residual plasma samples from blood donors who attended the Blood Bank Center at "Dr. Jose E. Gonzalez" University Hospital and the Blood Bank of the Transfusion Center of the Secretariat of Health of Nuevo Leon in Northeast Mexico during 2020. Each 
sample was identified with a numeric code for privacy protection. Sociodemographic data, hematic biometry values, and somatometric parameters were taken from the donor management database of each blood bank. All blood donors were aged 18-65 years and met the requirements of the Mexican Official Norm NOM-253-SSA1-2012.

During the donation protocol, all donors underwent a medical interview and were asked whether they had experienced any symptoms that suggested respiratory illness or any other infectious disease in the last 30 days. Donors with risk factors for bloodtransmitted diseases, recent disease, or who were deemed unhealthy during the physical examination were excluded. Once the donation was approved, blood was fractionated and the fresh plasma was kept at $-30{ }^{\circ} \mathrm{C}$ until use.

\subsection{Serological Analysis}

For antibody testing, we performed a chemiluminescent microparticle immunoassay (CMIA) for the qualitative detection of IgG antibodies against SARS-CoV-2 nucleoprotein using an anti-SARS-CoV-2 IgG kit (06R8620, from Abbott Laboratories, Abbott Park, IL, USA).

Plasma samples were run in an ARCHITECT system (from Abbott Diagnostics, Abbott Park, IL, USA following the manufacturer's instructions. This laboratory test was only used to determine past infection events. In this study, an index sample/calibrator (S/C) threshold of 1.4 or greater was taken as a positive result. FDA analysis reported sensitivity and specificity values of $90 \%$ and $100 \%$, respectively (data available in https: //www.accessdata.fda.gov/cdrh_docs/presentations/maf/maf3305-a001.pdf, accessed: 22 May 2021) and no significant cross-reactivity has been observed [20,21].

Serologic routine tests against Treponema pallidum (VDRL), hepatitis $\mathrm{C}$ virus (HCV), human immunodeficiency virus type 1 (HIV-1), hepatitis B virus (HBV), and Trypanosoma cruzi (Chagas disease) antibodies were run in an ARCHITECT i2000 SR analyzer (Abbott Diagnostics, Chicago, IL, USA). The presence of antibodies against Brucella was determined by the Rose Bengal Test (Licon, Mexico City, Mexico), a rapid slide-type agglutination assay.

\subsection{Data Analysis}

We tabulated the data in a Microsoft Excel ${ }^{\circledR}$ spreadsheet with donors' demographic characteristics (sex, age, blood type, and education level) and test results, reported by numeric code for privacy. The date of the donation was used as a reference to plot cases per epidemiological week (epi week), and the address was used for geographical mapping.

The raw SARS-CoV-2 prevalence in blood donors and the adjusted prevalence according to the sensitivity and specificity of the assay were calculated [22]. Prevalence was estimated as total prevalence (number of IgG-positive samples/total tested samples), weekly prevalence (number of IgG-positive samples/number of analyzed samples per epi week), and by donor demographic characteristics (IgG-positive samples/number of analyzed samples per group). According to the data type, we performed a $\chi^{2}$ test for categorical variables expressed as frequencies or percentages for descriptive statistics, using GraphPad Prism 9.1 software. To evaluate the association between SARS-CoV-2 infection and potential risk factors, we used logistic regression models and odds ratios (ORs). Statistical tests at a $5 \%$ significance level were adopted for relating the prevalence of anti-IgG antibodies to SARS-CoV-2 to donors' characteristics (sex, age group, blood type, and education level). Statistical analysis was performed using SPSS software.

The donors' home addresses were used to map the cases to the geographical distribution across Nuevo Leon using ArcGIS v10.2.2 (ESRI, Redlands, CA, USA). Official records of COVID-19 cases, confirmed by RT-qPCR (quantitative reverse-transcription PCR), were obtained from epidemiological reports available from https:/ / www.nl.gob. $\mathrm{mx}$ / publicaciones / casos-de-covid-19-en-nuevo-leon, last accessed 22 may 2021). Cases detected by RT-qPCR represent mainly symptomatic individuals who attended COVID-19 statal attention centers. 


\section{Results}

\subsection{Study Population}

A total of 2068 plasma samples from blood donors were obtained from 1 January to 15 December 2020. Males contributed $73.83 \%(1527 / 2068)$ of the plasma specimens, and $26.1 \%(541 / 2068)$ were from females. The median age of subjects studied was 34 years (IQR 16 years). The samples were grouped per epi week and divided into two observational periods: the pre-pandemic period (epi week 1 to 10) and the pandemic phase (epi week 11 to 52) (Figure 1A). Based on these criteria, 37 plasma samples were classified as pre-pandemic and 2031 as pandemic. This last subset was used for data analysis and seroprevalence estimation.

\subsection{Detection of Anti-SARS-CoV-2 IgG in Plasma Derived from Blood Donors}

All plasma samples from the pre-pandemic period tested negative for anti-SARS-CoV2 IgG. However, we detected 99 reactive plasma samples among the pandemic group, leading us to estimate a raw prevalence of $4.87 \%$ and $5.3 \%$ after adjusting prevalence for test specificity and sensitivity. Of the 99 positive cases, 65 were males (65.65\%) and 34 were females $(34.45 \%)$.

The main characteristics of the pandemic samples group and its specific prevalence values calculated for gender, age group, blood type, and education level are shown in Table S1. Despite the male-to-female ratio of 2.80:1, we observed that the incidence was lower in males $(4.34 \%)$ than females $(6.36 \%)(p=0.0436)$ (Figure 2, Table S2). Seroprevalence was 1.5 -fold higher among female donors compared to males. Persons with a higher education level were more exposed to SARS-CoV-2; prevalence in this group was $9.29 \%$ $(p<0.0001)$. While blood of types B $(5.04 \%)$ and $\mathrm{AB}(6.25 \%)$ was more likely to test positive, there was no significant difference from the other groups. Based on the clinical history and medical interviews, reactive plasma can be considered as evidence of asymptomatic SARS-CoV-2 infection.

\section{Sex}

Age group

Males Females

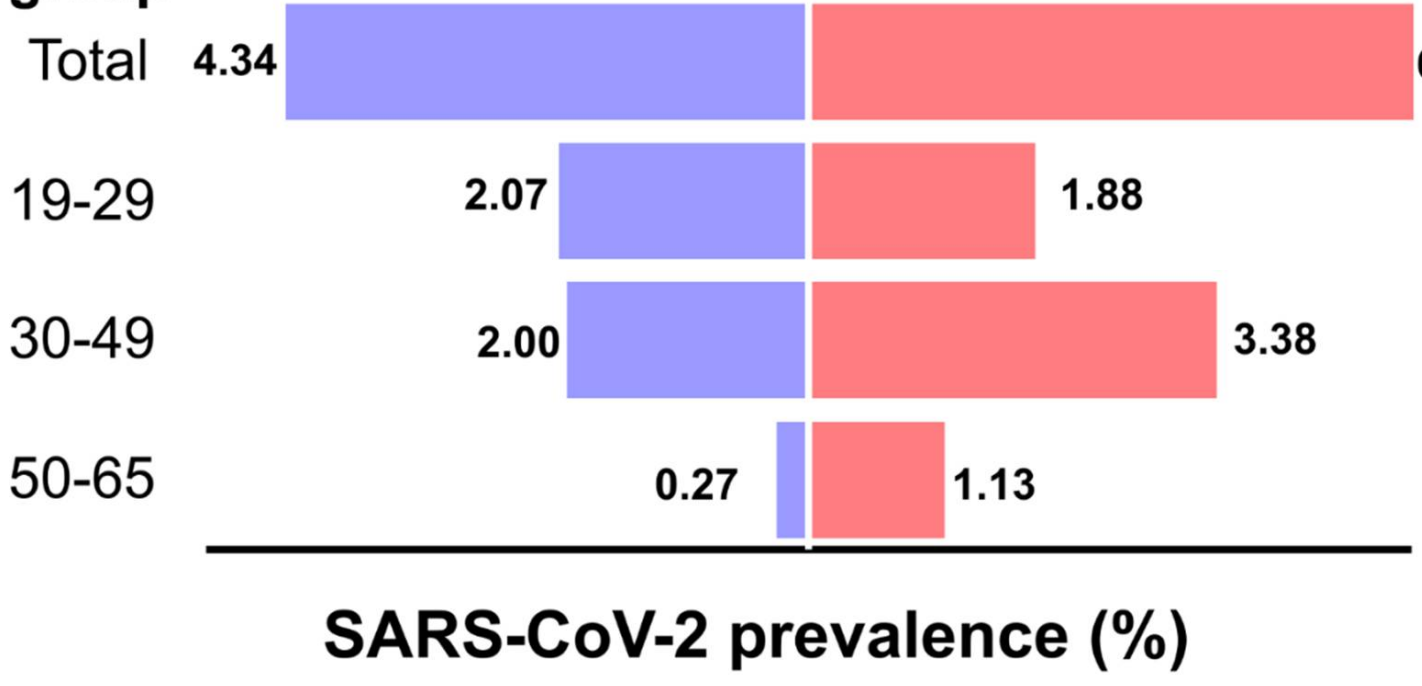

Figure 2. Sex and age distribution of SARS-CoV-2 IgG (+) samples. $n=99 / 2031$. 
Temporal distribution analyses showed that the first IgG-positive detection dated from 31 May, which was 12 weeks after the first confirmed COVID-19 case in Nuevo Leon (Figure 1B). We observed an increasing trend in weekly seroprevalence from $1.8 \%$ in May (epi week 23) to 19\% in August (epi week 33), to $27.59 \%$ by the end of the year (epi week 51). Asymptomatic case dynamics tended to be similar to the curve of weekly RTqPCR-confirmed cases reported by health instances, but in a delayed manner (Figure 1A).

Comparison of antibody titers showed no significant variation between antibody titers among reactive samples and different dates of collection (Figure 3).

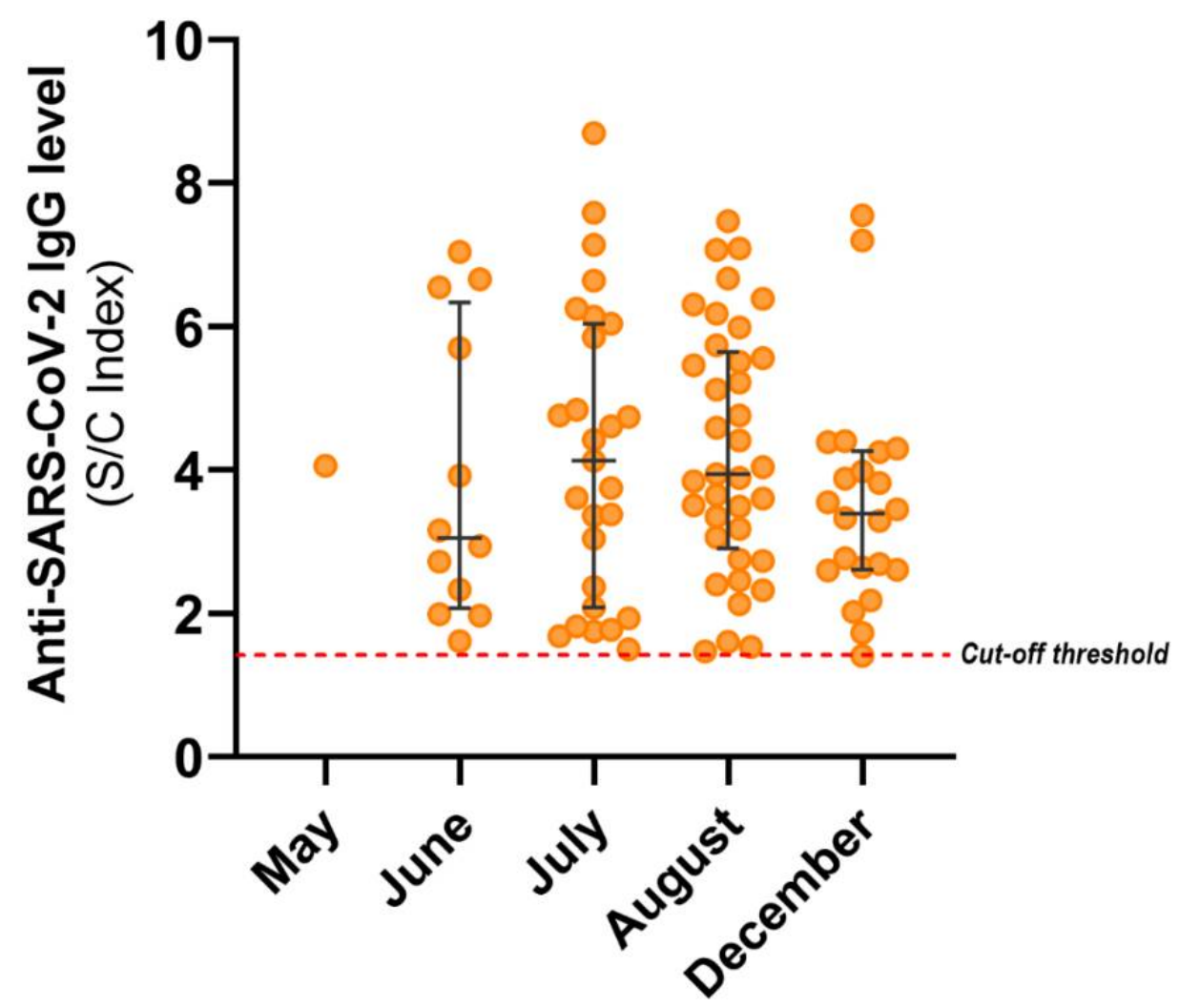

Figure 3. Anti-SARS-CoV-2 IgG titers across the collection period. S/C index values for the 99 reactive samples are graphed per month. Median and IQR are shown. Variation of antibody titers was not significant.

\subsection{Geographical Distribution of Observed Asymptomatic SARS-CoV-2 Infections}

Plasma samples were collected in 41/51 municipalities of Nuevo Leon (Table S3). The 99 SARS-CoV-2 IgG-positive cases observed were distributed across 21 of Nuevo Leon's municipalities, with Monterrey, Apodaca, and Escobedo contributing almost half (46.46\%) of the observed cases (Figure 4B).

The specific prevalence varied depending on the geographical zone. Inside the metropolitan area, specific region prevalence varied noticeably from $1.1 \%(1 / 92)$ in Santa Catarina to $6.5 \%(12 / 184)$ in Apodaca, but in rural areas, rates were higher and reached values over $30 \%$ (Figure 4 C). 
A Nuevo Leon location

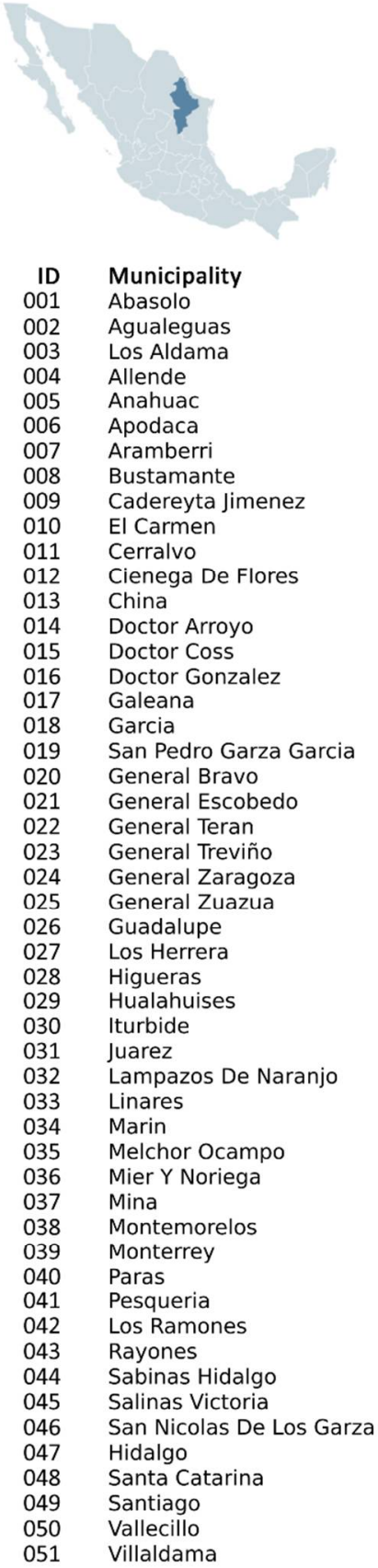

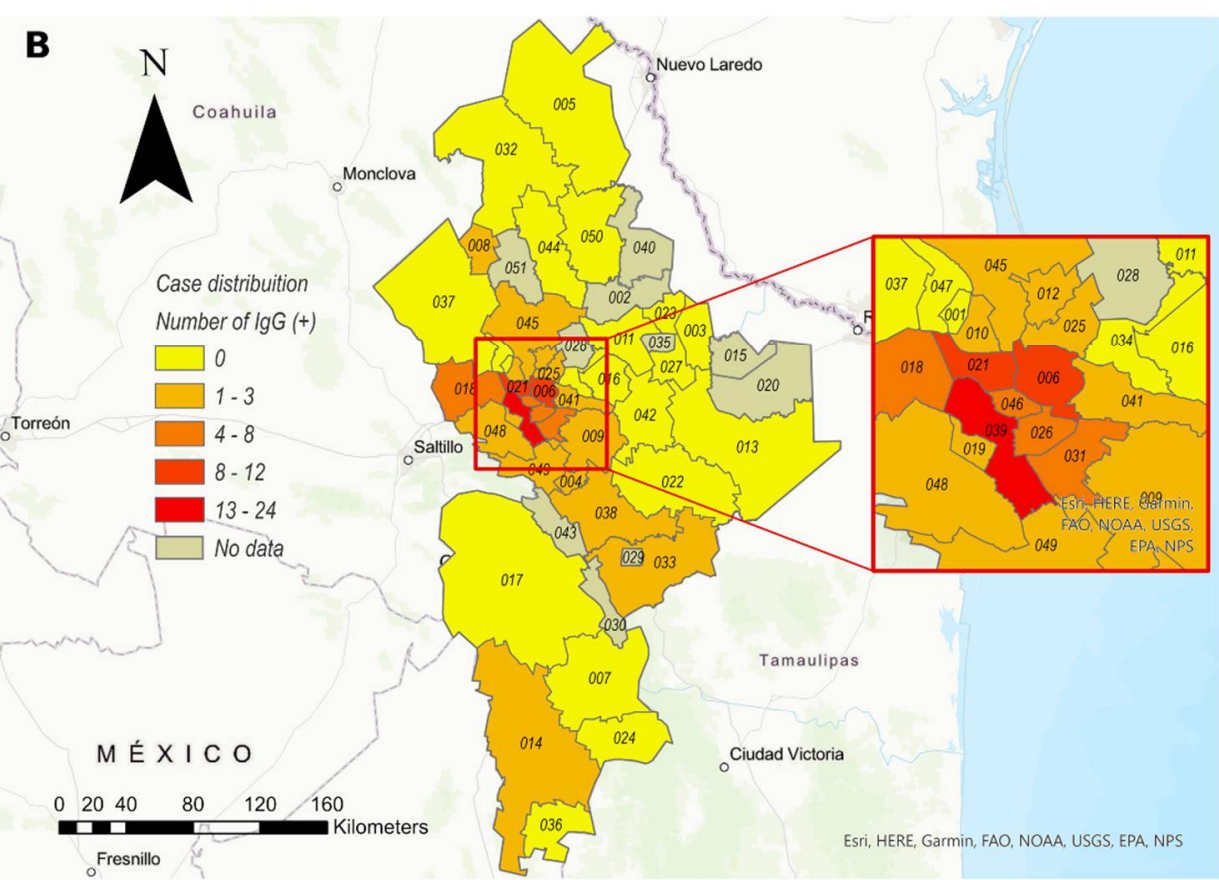

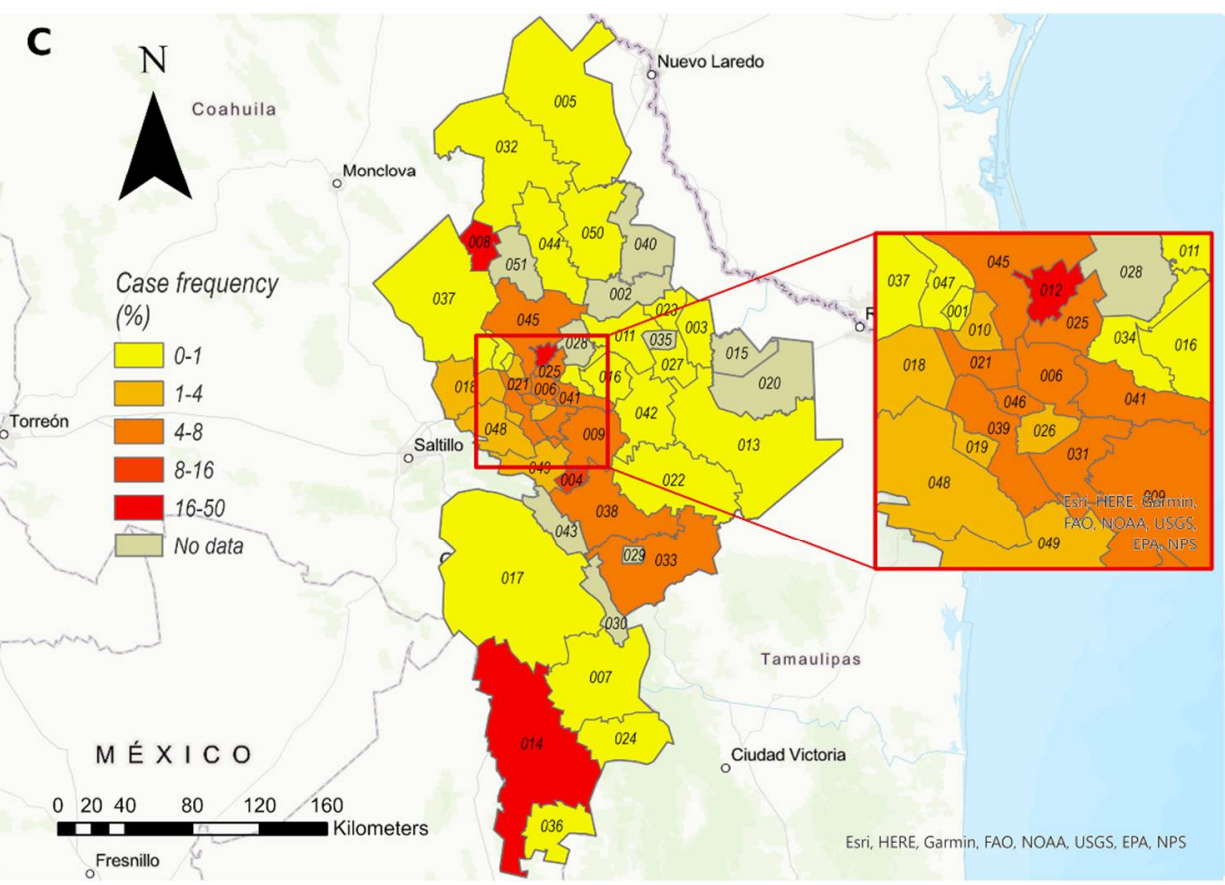

Figure 4. Geographical distribution of asymptomatic SARS-CoV-2 infection events determined by anti-SARS-CoV-2 IgG detection in the Nuevo Leon area. (A) Location of Nuevo Leon State in Mexico and municipalities. (B) Absolute number of cases per municipality detected during our study. (C) Proportion of cases to samples per municipality.

\section{Discussion}

It has been estimated that $15 \%$ to $46 \%$ of SARS-CoV- 2 infections are asymptomatic [23], but these rates can vary widely according to a population's variations of exposure over time, the epidemiological management of the pandemic, testing methods, and other stillunknown factors that affect different regions in the world.

Despite the expected fluctuation of the seroprevalence throughout the year, in this study we found a clear increase in asymptomatic SARS-CoV-2 cases in the studied population. During 2020, the prevalence increased from $1.8 \%$ (epi week 23) to $27.59 \%$ (epi 
week 51) with an average of 19\% (epi week 33) (Figure 1B). The increasing seroprevalence observed among the Nuevo Leon population indicates an important elevation in SARSCoV-2 exposure and contagion in our region. Due to the tested group consisting mainly of healthy subjects, IgG-positive cases can be classified as asymptomatic SARS-CoV-2 cases.

The results showed that asymptomatic SARS-CoV-2 infections were more frequent in females, contrary to males who tend to be symptomatic with higher morbidity and mortality [24]. This observation can be attributed to the fact that the innate antiviral immune response is higher in women [25].

Regarding the overall seroprevalence data reported here (4.87\% adjusted to $5.3 \%$ ), we found a higher prevalence than previously reported in blood donors in Wuhan, China with 2.29\% [26], the Netherlands with 2.7\% [15], and Denmark with 1.7\% [14] at the beginning of the pandemic. In a similar study, the American Red Cross in the United States tested all blood donations from June to August 2020 for anti-SARS-CoV-2 IgG antibodies and found that $1.82 \%(17,336 / 953,926)$ of donations were positive [27]. We also found, as in other regions, that anti-SARS-CoV-2 seroprevalence in blood donors initially grew slowly and then rapidly, but varied by region, as demonstrated by a report from the United Kingdom [28]. Similarly, analysis of the distribution of samples showed that most of these samples were from urban areas and regions with a high population density.

Until now, there have been few studies on the prevalence of SARS-CoV-2 in Mexico. One of them was focused on a specific area (Guadalupe, a municipality in Nuevo Leon) [19], while the other was conducted in another state (Veracruz State in Southeast Mexico) [18]. Both involved cross-sectional analyses during July 2020. Prevalence among asymptomatic subjects from Guadalupe, Nuevo Leon $(4.15 \%, 114 / 2741)$ was within the reported rates $(1.6-9.2 \%)$ for the same period. In contrast, values observed in Nuevo Leon differed greatly from those observed in Veracruz, where $21 \%$ of healthy subjects tested positive, showing that the pandemic evolved differently in each city, and control measures needed to be adapted accordingly.

Despite the low risk of SARS-CoV-2 in blood transfusions [29], the prevalence of this new viral agent was higher than other infectious diseases commonly analyzed in blood donors (Figure S1). SARS-CoV-2 increased over time while infections caused by Brucella spp, HCV, HIV-1, Trypanosoma cruzi, and Treponema pallidum remained similar to previous reports in the region [30,31].

Titers of SARS-CoV-2-specific antibodies can remain elevated and stable for up to five months [13]. In this study, samples were collected continuously during the five months after diagnosis of the first confirmed case by RT-qPCR in Nuevo Leon. For this reason, results are unlikely to be affected by a drop in SARS-CoV-2 antibody levels. In addition, we performed a second collection in December to determine if antibody level distribution had changed in a significant manner since August; antibody levels among positive cases remained similar (Figure 3). However, future seroprevalence studies need to be done to assess the lifespan of the antibodies.

As for the limitations of our study, first, we note that the estimated seroprevalence may not reflect the true underlying proportion of those exposed to SARS-CoV-2 in our country because blood donors are not representative of the overall population; second, we did not perform virus neutralization assays, therefore the neutralizing activity of the detected IgG antibodies is unknown; third, a rapid decline in antibody titers and pro-inflammatory cytokines may be a common feature of non-severe SARS-CoV-2 infection, suggesting that asymptomatic individuals may have a weaker immune response to SARS-CoV-2 infection in contrast to symptomatic subjects [32]; fourth, at the time of this work, due to government regulations in Mexico, SARS-CoV-2 serological tests available for diagnostics were limited to IgG analysis. For this reason, we only detected infections with at least two or three weeks of evolution; more recent infections may have gone unnoticed, meaning prevalence could be higher.

Finally, the results of this study allow us to highlight two important points: there is a growing trend of seroprevalence over time, parallel to the constantly increasing epidemic 
curve in our region, and the higher prevalence of positive plasma found in female subjects under 49 years of age is associated with asymptomatic infection in these donors. This work demonstrated that screening blood donors for SARS-CoV-2 antibodies strengthens existing evidence that this group can be used as a sentinel population to track the progression of the COVID-19 pandemic.

Supplementary Materials: The following are available online at https://www.mdpi.com/article/10 .3390/v13071225/s1, Figure S1: Comparison of prevalence of SARS-CoV-2 IgG antibodies observed against other blood-borne infectious diseases during the same period, Table S1:Blood donor's characteristics and crude prevalence of antibodies to SARS-CoV-2 in the collected samples from Nuevo Leon Area, Mexico, during the pandemic period (epi week 11 to 51 of 2020),Table S2: Variable association to SARS-CoV-2 risk of infection, Table S3: Geographical distribution of tested samples across Nuevo Leon region and COVID-19 positivity ratio observed.

Author Contributions: All included authors met the ICMJE criteria for authorship and medical publication. All authors provided results, data collection, statistical analysis, and/or critical feedback for writing and intellectual manuscript design, and all authors approved the final version of the manuscript. Conceptualization, A.M.R.-E.; data curation, D.M.A.-N., D.R.R.-R., C.G.M.-L., S.A.-d.-1.-C., E.C.-P. and D.A.-S.; formal analysis, S.A.-d.-1.-C., E.C.-P. and G.R.P.-R.; investigation, N.M.-A., D.M.A.-N., D.R.R.-R., C.G.M.-L. and G.R.P.-R.; methodology, N.M.-A., D.M.A.-N., D.R.R.-R., C.G.M.-L., S.A.-d.-1.-C., E.C.-P., E.A.D.-C. and E.G.-G.; project administration, N.M.-A., E.A.D.-C., R.C.-T., F.P.-C., D.A.-S., A.F.-A. and R.M.-d.-O.-L.; resources, N.M.-A., K.A.G.-H. and S.A.L.-S.; software, K.A.G.-H.; supervision, E.A.D.-C., R.C.-T., F.P.-C., S.A.L.-S., A.F.-A., J.R.-J., A.B.L.-G. and A.M.R.-E.; validation, E.G.-G. and A.B.L.-G.; writing—original draft, N.M.-A. and A.M.R.-E.; writingreview and editing, K.A.G.-H., R.C.-T., S.A.L.-S., D.A.-S., G.R.P.-R., J.R.-J., E.G.-G., R.M.-d.-O.-L., C.T.-G., A.M.R.-E. and M.E.d.-1.-O.-C. All authors have read and agreed to the published version of the manuscript.

Funding: This research was funded by CONACYT Grants: 312135 to N.M.-A.; 314867 toA.M.R.-E., 312368 to S.A.L.-S., and 312328 to K.A.G.-H.

Institutional Review Board Statement: This study was approved by the Institutional Review Board of School of Medicine and "Dr. Jose E. Gonzalez" University Hospital (BI21-00001) and the Health Secretariat of Nuevo Leon (DEISC-287 190120024).

Informed Consent Statement: Due to the use of exclusively residual material in this study, the need for informed consent was waived.

Data Availability Statement: All primary data presented in this study are available from the corresponding author upon reasonable request. Primary data exist for all figures.

Acknowledgments: We thank the staff of the Central Blood Bank of the Department of Clinical Pathology, "Jose E. Gonzalez" University Hospital and School of Medicine, UANL; and the Secretariat of Health of Nuevo Leon (Transfusion Center CETS) for their technical help and facilitating this study. We also thank Sergio Lozano Rodriguez for his critical reading and editing of the manuscript.

Conflicts of Interest: The authors declare no conflict of interest.

\section{References}

1. Johns Hopkins University \& Medicine. Coronavirus Resource Center. Available online: https://coronavirus.jhu.edu/map.html (accessed on 22 May 2021).

2. Secretaría de Salud de Nuevo Leon. Casos de COVID-19 en Nuevo León. Available online: https://www.nl.gob.mx/ publicaciones / casos-de-covid-19-en-nuevo-leon (accessed on 22 May 2021).

3. INEGI. Número de Habitantes Nuevo Leon. Available online: http://cuentame.inegi.org.mx/monografias/informacion/NL/ Poblacion/default.aspx?tema=ME\&e=19 (accessed on 22 May 2021).

4. The epidemiological characteristics of an outbreak of 2019 novel coronavirus diseases (COVID-19) in China. Zhonghua Liu Xing Bing Xue Za Zhi 2020, 41, 145-151.

5. Dong, Y.; Mo, X.; Hu, Y.; Qi, X.; Jiang, F.; Jiang, Z.; Tong, S. Epidemiology of COVID-19 Among Children in China. Pediatr. 2020, 145, e20200702. [CrossRef]

6. Mayor, S. Covid-19: Nine in 10 pregnant women with infection when admitted for delivery are asymptomatic, small study finds. BMJ 2020, 369, m1485. [CrossRef] [PubMed] 
7. Al-Sadeq, D.W.; Nasrallah, G.K. The incidence of the novel coronavirus SARS-CoV-2 among asymptomatic patients: A systematic review. Int. J. Infect. Dis. 2020, 98, 372-380. [CrossRef] [PubMed]

8. Lee, S.; Kim, T.; Lee, E.; Lee, C.; Kim, H.; Rhee, H.; Park, S.Y.; Son, H.-J.; Yu, S.; Park, J.W.; et al. Clinical Course and Molecular Viral Shedding Among Asymptomatic and Symptomatic Patients With SARS-CoV-2 Infection in a Community Treatment Center in the Republic of Korea. JAMA Intern. Med. 2020, 180. [CrossRef] [PubMed]

9. Huang, L.; Zhang, X.; Zhang, X.; Wei, Z.; Zhang, L.; Xu, J.; Liang, P.; Xu, Y.; Zhang, C.; Xu, A. Rapid asymptomatic transmission of COVID-19 during the incubation period demonstrating strong infectivity in a cluster of youngsters aged 16-23 years outside Wuhan and characteristics of young patients with COVID-19: A prospective contact-tracing study. J. Infect. 2020, 80, e1-e13. [CrossRef]

10. Long, Q.X.; Liu, B.Z.; Deng, H.J.; Wu, G.C.; Deng, K.; Chen, Y.K.; Liao, P.; Qiu, J.; Lin, Y.; Huang, A.L.; et al. Antibody responses to SARS-CoV-2 in patients with COVID-19. Nat. Med. 2020, 26, 845-848. [CrossRef]

11. Chen, Y.; Li, L. SARS-CoV-2: Virus dynamics and host response. Lancet Infect. Dis. 2020, 20, 515-516. [CrossRef]

12. Okba, N.M.A.; Müller, M.A.; Li, W.; Wang, C.; GeurtsvanKessel, C.H.; Corman, V.M.; Lamers, M.M.; Sikkema, R.S.; De Bruin, E.; Chandler, F.D.; et al. Severe Acute Respiratory Syndrome Coronavirus 2-Specific Antibody Responses in Coronavirus Disease Patients. Emerg. Infect. Dis. 2020, 26, 1478-1488. [CrossRef]

13. Wajnberg, A.; Amanat, F.; Firpo, A.; Altman, D.R.; Bailey, M.J.; Mansour, M.; McMahon, M.; Meade, P.; Rao Mendu, D.; CordonCardo, C.; et al. Robust neutralizing antibodies to SARS-CoV-2 infection persist for months. Science 2020, 370, 1227-1230. [CrossRef]

14. Erikstrup, C.; Hother, C.E.; Pedersen OB, V.; Mølbak, K.; Skov, R.L.; Holm, D.K.; Sækmose, S.G.; Nilsson, A.C.; Brooks, P.T.; Ullum, H.; et al. Estimation of SARS-CoV-2 Infection Fatality Rate by Real-time Antibody Screening of Blood Donors. Clin. Infect. Dis. 2021, 72, 249-253. [CrossRef]

15. Slot, E.; Hogema, B.M.; Reusken, C.B.; Reimerink, J.H.; Molier, M.; Karregat, J.H.; Ijlst, J.; Novotný, V.M.J.; van Lier, R.A.W.; Zaaijer, H.L. Herd immunity is not a realistic exit strategy during a COVID-19 outbreak. Nat. Portf. 2020. [CrossRef]

16. Ng, D.L.; Goldgof, G.M.; Shy, B.R.; Levine, A.G.; Balcerek, J.; Bapat, S.P.; Prostko, J.; Rodgers, M.; Coller, K.; Chiu, C.Y.; et al. SARS-CoV-2 seroprevalence and neutralizing activity in donor and patient blood. Nat. Commun. 2020, 11, 4698. [CrossRef] [PubMed]

17. Uyoga, S.; Adetifa, I.M.; Karanja, H.K.; Nyagwange, J.; Tuju, J.; Wanjiku, P.; Aman, R.; Mwangangi, M.; Amoth, P.; Warimwe, G.M.; et al. Seroprevalence of anti-SARS-CoV-2 IgG antibodies in Kenyan blood donors. Science 2021, 370, 1227-1230. [CrossRef] [PubMed]

18. Remes-Troche, J.M.; Ramos-De-la-Medina, A.; Manríquez-Reyes, M.; Maldonado LM, P.; Solis-Gonzalez, M.A.; Hernandez-Flores, K.; Vivanco-Cid, H. Prevalence of SARS-CoV-2 IgG antibodies in a population from Veracruz (Southeastern Mexico). medRxiv 2020. [CrossRef]

19. Díaz-Salazar, C.; Sánchez-García, A.; Rodríguez-Gutiérrez, R.; Camacho-Ortiz, A.; Saldívar-Rodríguez, D.; González-González, J.G. Prevalence and associated characteristics of anti-SARS-CoV-2 antibodies in Mexico 5 months after pandemic arrival. Res. Sq. 2020. [CrossRef]

20. Manalac, J.; Yee, J.; Calayag, K.; Nguyen, L.; Patel, P.M.; Zhou, D.; Shi, R.Z. Evaluation of Abbott anti-SARS-CoV-2 CMIA IgG and Euroimmun ELISA IgG/IgA assays in a clinical lab. Clin. Chim. Acta 2020, 510, 687-690. [CrossRef]

21. Meschi, S.; Colavita, F.; Bordi, L.; Matusali, G.; Lapa, D.; Amendola, A.; Vairo, F.; Ippolito, G.; Capobianchi, M.R.; Castilletti, C.; et al. Performance evaluation of Abbott ARCHITECT SARS-CoV-2 IgG immunoassay in comparison with indirect immunofluorescence and virus microneutralization test. J. Clin. Virol. 2020, 129, 104539. [CrossRef]

22. Sempos, C.T.; Tian, L. Adjusting Coronavirus Prevalence Estimates for Laboratory Test Kit Error. Am. J. Epidemiol. 2021, 190, 109-115. [CrossRef]

23. Rabaan, A.A.; Al-Ahmed, S.H.; Haque, S.; Sah, R.; Tiwari, R.; Malik, Y.S.; Dhama, K.; Yatoo, M.I.; Bonilla-Aldana, D.K.; Rodriguez-Morales, A.J.; et al. SARS-CoV-2, SARS-CoV, and MERS-COV: A comparative overview. Infez. Med. 2020, 28, 174-184. [PubMed]

24. Nimgaonkar, I.; Valeri, L.; Susser, E.; Hussain, S.; Sunderram, J.; Aviv, A. The age pattern of the male-to-female ratio in mortality from COVID-19 mirrors that of cardiovascular disease in the general population. Aging 2021, 13, 3190-3201. [CrossRef]

25. Gupta, S.; Nakabo, S.; Blanco, L.P.; O’Neil, L.J.; Wigerblad, G.; Goel, R.R.; Mistry, P.; Jiang, K.; Carmona-Rivera, C.; Chan, D.W.; et al. Sex differences in neutrophil biology modulate response to type I interferons and immunometabolism. Proc. Natl. Acad. Sci. USA 2020, 117, 16481-16491. [CrossRef] [PubMed]

26. Chang, L.; Hou, W.; Zhao, L.; Zhang, Y.; Wang, Y.; Wu, L.; Xu, T.; Wang, J.; Ma, J.; Wang, L.; et al. The prevalence of antibodies to SARS-CoV-2 among blood donors in China. Nat. Commun. 2021, 12, 1383. [CrossRef]

27. Dodd, R.Y.; Xu, M.; Stramer, S.L. Change in Donor Characteristics and Antibodies to SARS-CoV-2 in Donated Blood in the US, June-August 2020. JAMA 2020, 324, 1677-1679. [CrossRef] [PubMed]

28. Thompson, C.P.; Grayson, N.E.; Paton, R.S.; Bolton, J.S.; Lourenço, J.; Penman, B.S.; Lee, L.N.; Odon, V.; Mongkolsapaya, J.; Chinnakannan, S.; et al. Detection of neutralising antibodies to SARS coronavirus 2 to determine population exposure in Scottish blood donors between March and May 2020. Eurosurveillance 2020, 25, 1-9. [CrossRef] [PubMed] 
29. Cappy, P.; Candotti, D.; Sauvage, V.; Lucas, Q.; Boizeau, L.; Gomez, J.; Enouf, V.; Chabli, L.; Pillonel, J.; Laperche, S.; et al. No evidence of SARS-CoV-2 transfusion transmission despite RNA detection in blood donors showing symptoms after donation. Blood 2020, 136, 1888-1891. [CrossRef] [PubMed]

30. Rodríguez Rodríguez, D.; Garza Rodríguez, M.; Chavarria, A.M.; Ramos-Jiménez, J.; Rivera, M.A.; Taméz, R.C.; Farfan-Ale, J.; Rivas-Estilla, A.M. Dengue virus antibodies in blood donors from an endemic area. Transfus. Med. 2009, 19, 125-131. [CrossRef] [PubMed]

31. Arellanos-Soto, D.; Mendoza-Tavera, N.; Ramos-Jiménez, J.; Cázares-Taméz, R.; Ortega-Soto, A.; Rivas-Estilla, A.M. Constant risk of dengue virus infection by blood transfusion in an endemic area in Mexico. Transfus. Med. 2015, 25, 122-124. [CrossRef]

32. Long, Q.X.; Tang, X.J.; Shi, Q.L.; Li, Q.; Deng, H.J.; Yuan, J.; Hu, J.L.; Xu, W.; Zhang, Y.; Huang, A.L.; et al. Clinical and immunological assessment of asymptomatic SARS-CoV-2 infections. Nat. Med. 2020, 26, 1200-1204. [CrossRef] 